\title{
Effet de la thérapie visuelle sur les mesures de la fonction oculomotrice chez les patients atteints du syndrome de post-commotion
}

\author{
Paul Rollett, OD, FCOVD \\ Okanagan Vision Therapy \\ Optometry
}

Garrett Morandi, Ph. D.

Université de la Saskatchewan

\begin{abstract}
Résumé
La dysfonction oculomotrice est un symptôme courant du syndrome de post-commotion (SPC). Dans cette étude, on a étudié rétrospectivement l'efficacité de la thérapie visuelle (TV) pour le traitement des symptômes liés au SPC par rapport au système visuel. Dans l'ensemble, 56 patients ont été sélectionnés, qui présentaient tous une déficience sur le plan clinique d'au moins une mesure du fonctionnement oculomoteur. Les activités liées à la TV étaient grandement variées et établies au cas par cas, mais elles visaient toutes à améliorer l'une des cinq principales fonctions visuelles. À la suite de la TV, tous les patients ont démontré des améliorations statistiquement ou cliniquement significatives, comme définies par l'utilisation des normes de Morgan, dans au moins une mesure du fonctionnement oculomoteur lié au SPC. En général, les améliorations les plus importantes des mesures du fonctionnement oculomoteur concernaient le point de convergence proche, la flexibilité de vergence et la flexibilité d'accommodation. Les patients qui ont suivi 20 séances de TV avaient de meilleurs résultats (aussi moins variables) que ceux qui ont suivi entre 5 et 10 séances. De plus, la TV a diminué les symptômes d'inconfort visuel chez les patients qui présentaient un SPC. Les résultats de cette analyse rétrospective démontrent des améliorations importantes dans les résultats mesurés pour tous les patients qui ont suivi une TV et appuient la TV comme option de traitement pour les symptômes du SPC.
\end{abstract}

\section{INTRODUCTION}

Un traumatisme crânien léger (TCL), comme celui attribuable à un coup à la tête, à un tremblement violent ou à un mouvement de la tête ou du corps, peut entraîner des symptômes de commotion cérébrale. ${ }^{1}$ Après une blessure, les symptômes d'une commotion cérébrale se manifestent généralement au cours des 7 à 10 premiers jours et peuvent persister jusqu'à un an ou plus. Les symptômes d'une commotion cérébrale sont collectivement appelés un syndrome post-commotionnel (SPC). ${ }^{1}$ Les symptômes du SPC comprennent, sans s'y limiter, les maux de tête, les étourdissements, la fatigue, la sensibilité au bruit et à la lumière, ainsi que les problèmes liés au système visuel. En particulier, entre $11 \%$ et $54 \%$ des patients des études de cohorte de soldats ou de patients d'accidents automobiles avec des symptômes de TCL ont reçu un diagnostic d'une ou de plusieurs dysfonctions oculomotrices, y compris une insuffisance de convergence, une insuffisance d'accommodation, une détérioration du mouvement de version et des problèmes mineurs d'alignement oculaire. ${ }^{2-10}$ De plus, $46 \%$ des adolescents présentant des symptômes de commotion cérébrale avaient plus d'un diagnostic lié à la vision, comme un trouble d'accommodation, une insuffisance de convergence ou une dysfonction saccadique. ${ }^{11}$ L'insuffisance de convergence est un trouble de la vision prévalent caractérisé par la réduction de l'amplitude de convergence de fusion et un point de convergence diminué, qui peut se manifester sous forme de vision floue, de fatigue oculaire et de maux de tête dans un travail de près. ${ }^{12-14}$ Des travaux antérieurs ont suggéré 
que les symptômes d'insuffisance de convergence et les problèmes oculomoteurs connexes sont probablement dus à une compensation de convergence excessive pour les grandes postures exophoriques dans la vision de proche. ${ }^{15}$

La thérapie visuelle optométrique (TV) est devenue une option de traitement prometteuse pour les patients qui présentent des symptômes de SPC ou de TCL.,5 Par exemple, dans une étude rétrospective, 90 \% des participants ayant suivi une TV pour une lésion cérébrale acquise ont connu une amélioration complète ou importante d'au moins un des symptômes notés de lésion cérébrale acquise, et ces améliorations ont persisté quand elles ont été mesurées deux mois ou plus après la TV. ${ }^{16}$ En outre, des améliorations similaires ont été observées dans les mesures du fonctionnement oculomoteur chez la plupart des patients ayant suivi une TV pour un SPC ou un TCL.,5,17-20 De plus, il a été démontré que la TV améliore les mesures de la concentration, de la mémoire et de la compréhension chez les patients qui présentent des symptômes de SPC ou de TCL. ${ }^{20}$ En ayant recours au sondage sur les symptômes pendant l'essai du traitement de l'insuffisance de convergence (appelé CISS, en anglais), Alvarez et al. ont montré des améliorations importantes chez les patients avec une insuffisance de convergence après 18 heures de formation de vergence, et leurs scores étaient semblables à ceux des patients témoins. En outre, les travaux d'Alvarez et al. appuient davantage la notion selon laquelle l'insuffisance de convergence est causée par une réduction significative de la vitesse de convergence à son apogée, et la notion selon laquelle l'entraînement de vergence mène à une augmentation de l'activité fonctionnelle. Par ailleurs, Scheiman et Wick. ont démontré que la vergence au bureau et la thérapie de l'accommodation à domicile peuvent entraîner une réduction durable des symptômes d'insuffisance de convergence.

Bien qu'il existe des données probantes appuyant la TV dans le traitement des symptômes liés au SPC ou liés aux traumatismes crâniens légers, des données supplémentaires soutenant le rôle positif de la TV dans le traitement des traumatismes cérébraux seraient opportunes. ${ }^{4,22}$ Par conséquent, dans le cadre de ces travaux, nous présentons les résultats d'une analyse rétrospective de l'efficacité du traitement de la thérapie visuelle pour améliorer diverses fonctions oculomotrices chez les patients qui présentent des symptômes du SPC.

\section{MÉTHODES}

Les patients ont donné leur consentement exprès avant le début du traitement de la dysfonction oculomotrice.

\section{ÉVALUATION DU PATIENT}

Les patients chez qui un médecin a diagnostiqué un SPC ont fait l'objet d'une évaluation oculovisuelle approfondie. Les tests de vision pertinents dans le cadre de cette étude comprennent l'évaluation du point de convergence proche (PCP), la flexibilité de vergence (base vers l'extérieur = 12 et base vers l'intérieur = 3), la flexibilité d'accommodation en ayant recours à une puissance de lentille de + / - 2,00 D (selon l'âge), les mesures de la vision stéréoscopique de près $(40 \mathrm{~cm}$ ) et à distance (6 mètres) à l'aide de l'évaluation Randot, du King-Devick test, des amplitudes de fusion positives de vergence de près $(40 \mathrm{~cm})$ et à distance (6 mètres), des amplitudes de fusion négatives de vergence de près $(40 \mathrm{~cm})$ et à distance (6 mètres) et de l'achèvement du sondage CISS avant le traitement, puis également une semaine après. Tous les essais ont été effectués de la façon décrite par Elliott. ${ }^{23}$ Les patients présentant des paralysies nerveuses, des restrictions des muscles extra-oculaires, une pression intraoculaire élevée ou une perte globale du champ visuel déterminée par la confrontation ont été exclus de l'étude.

\section{RÉGIME DE TRAITEMENT DE LA THÉRAPIE VISUELLE}

Après l'évaluation, pour chaque patient, le nombre de séances requises a été déterminé selon notre meilleur jugement, la littérature antérieure et, parfois en fonction de l'approbation des tiers payeurs. ${ }^{2,15,24}$ La thérapie visuelle comprenait des séances hebdomadaires d'une heure effectuée à la clinique et réalisées par des thérapeutes de la vision formés, ainsi que des séances quotidiennes de 15 minutes à mettre en pratique à la maison. Les activités prescrites étaient variées et établies au cas par cas. Néanmoins, elles visaient toutes à améliorer l'un des cinq principaux piliers de la fonction visuelle : l'intégration de la vision centrale-périphérique (relation spatiale), la coordination interoculaire (convergence et divergence), la focalisation oculaire (accommodation), la poursuite oculaire (poursuite et saccades douces) et l'interprétation des mouvements (suivi des objets en mouvement dans une scène complexe). Les détails des procédures utilisées au cours du traitement ont été précédemment décrits. ${ }^{15,25}$

\section{ANALYSE STATISTIQUE}

Des données sur les patients ont été recueillies entre le $1^{\mathrm{er}}$ janvier 2017 et le 31 décembre 2017 auprès de tous les patients recevant une thérapie visuelle pour un SPC $(n=56)$. On a établi une moyenne des scores individuels des 56 patients pour l'ensemble des résultats mesurés et on a comparé les valeurs avant (pré) et après (post) l'intervention de TV. Les résultats des patients pré et post TV ont été comparés selon l'utilisation du t-test dépendant $(\mathrm{p}<0,05)$. Les paramètres qui ne 
répondaient pas aux hypothèses de normalité ou de variance égale ont été comparés à l'aide du test de Wilcoxon, comme l'ont suggéré Rosner et al. ${ }^{26}$ Pour évaluer l'importance clinique des résultats pour les patients, les valeurs mesurées ou enregistrées ont été comparées à la documentation pertinente, à savoir le tableau des normes de Morgan. ${ }^{15}$

\section{RÉSULTATS}

\section{Aperçu des données}

Dans l'ensemble, 56 patients ont été inclus dans l'analyse rétrospective et les statistiques descriptives sont présentées au tableau 1. Les patients ont vécu toute une gamme d'événements qui ont entraîné un SPC, y compris des accidents automobiles dans 38 cas, des blessures sportives dans 10 cas et des chutes dans 8 cas. L'âge des patients variait entre 12 et 62 ans (écart-type moyen; $42,6 \pm 13,8$ ) et les patients ont suivi entre 5 et 20 séances de TV $(13,8 \pm 4,70)$ pour traiter les symptômes du SPC. La durée moyenne du programme pour les patients (15,2 semaines) était supérieure au nombre de séances de TV (13,8 séances) et reflète l'incidence des annulations de rendez-vous des patients ou des retards de traitement d'une semaine à l'autre sur la durée du programme. De plus, les patients ont généralement commencé un traitement de TV un mois après l'évaluation.

\section{Résultats mesurés chez les patients}

En général, le traitement des symptômes du SPC avec TV a entraîné des améliorations importantes de toutes les fonctions oculomotrices mesurées (tableau 2). Tous les patients inclus dans cette étude ont montré une amélioration d'au moins une mesure du fonctionnement oculomoteur. Quarante-six patients présentaient un PCP déficient sur le plan clinique et, à la suite de la TV, $86 \%$ des patients présentaient des améliorations significatives sur le plan clinique (tableau 2). Cinquante-cinq patients avaient une flexibilité de vergence déficiente sur le plan clinique (tableau 2) et, après la TV, tous les patients présentaient une amélioration de celle-ci, qui était significative sur le plan clinique dans 36 cas (65\%). En ce qui a trait à l'évaluation de la flexibilité d'accommodation, 20 des 22 patients avaient une déficience pertinente sur le plan clinique et, après le traitement, $90 \%$ des patients ont connu des améliorations cliniquement significatives. Lorsqu'ils ont été mesurés à l'aide du King-Devick test, 43 patients avaient un mouvement saccadique déficient sur le plan clinique et, après le traitement, 26 patients (60\%) ont connu des améliorations cliniquement significatives (tableau 2).

Quarante-six patients présentaient une déficience de stéréopsie pertinente sur le plan clinique lorsque testés à distance. Tous les patients, sauf 3 , ont vu leur note moyenne s'améliorer de $50 \%$, et ces améliorations étaient pertinentes sur le plan clinique dans 23 cas (50\%). Lorsque la stéréopsie a été évaluée à des distances rapprochées, 40 patients ont montré une déficience pertinente sur le plan clinique. Après le traitement, on a observé des améliorations moyennes de $45 \%$, et 30 patients ( $75 \%$ ) ont obtenu des résultats cliniquement significatifs. Cinquante-trois patients ont obtenu des scores supérieurs à 22 dans le sondage CISS sur les symptômes. Après le traitement, tous les patients ont connu une amélioration du score moyen de $48 \%$, et celles-ci étaient cliniquement significatives dans 35 cas (66\%) (tableau 2).

\section{RÉSULTATS DES TESTS DE CONVERGENCE ET DE DIVERGENCE CHEZ LES PATIENTS}

Les amplitudes de fusion de vergence positive et négative (FVP/FVN) ont été évaluées à distance (6 mètres) et de près $(40 \mathrm{~cm})$. Lorsqu'on a mesuré la FVP à distance, 51 patients présentaient une rupture de convergence déficiente sur le plan clinique. Quarante-neuf patients connaissaient une amélioration moyenne de $11 \mathrm{D}$ des scores de rupture fusionnelle positifs, et ces améliorations étaient pertinentes sur le plan clinique dans 26 cas (53 \%). Lorsqu'on a évalué le rétablissement de la FVP à distance, 46 patients obtenaient des scores de déficience sur le plan clinique. Après le traitement, tous les patients sauf un avaient amélioré leurs scores, et ces améliorations étaient cliniquement significatives pour 32 cas (69\%) (tableau 2). Lorsqu'on a évalué la FVN à distance, 52 patients avaient une déficience pertinente sur le plan clinique en matière de rupture de divergence. Après le traitement, 47 patients connaissaient une amélioration moyenne de $4,7 \mathrm{D}$, et cette amélioration était cliniquement significative pour 35 cas (67\%). Lorsqu'on a évalué le rétablissement de la FVN à distance, 42 patients obtenaient des scores de déficience pertinente sur le plan clinique. Tous les patients sauf un ont connu une amélioration des scores après la TV, et l'amélioration était cliniquement significative dans 40 cas (95\%) (tableau 2).

Des améliorations semblables à celles observées à distance ont été observées pour les amplitudes de fusion de vergence à proximité. Cinquante patients avaient une rupture de la FVP déficiente sur le plan clinique. Quarante-neuf patients présentaient des scores moyens améliorés de 19,6 D, et ces améliorations étaient pertinentes sur le plan clinique dans 46 cas (94\%). Lorsqu'on a évalué le rétablissement de la FVP à proximité, 47 patients obtenaient des scores de déficience pertinente sur le plan clinique. Dans l'ensemble, les patients ont constaté une amélioration moyenne de 18,5 D, et ces améliorations étaient pertinentes sur le plan clinique dans 42 cas (89 \%) (tableau 2). Lorsqu'on a évalué les amplitudes de FVN à proximité, 55 patients ont obtenu des déficits pertinents sur le plan 
clinique. Cinquante-trois patients ont vu une amélioration moyenne de 10,4 D, et ces améliorations étaient cliniquement significatives dans 30 cas (55\%). Lorsqu'on a évalué le rétablissement de la FVN à proximité, 45 patients obtenaient des scores de déficience pertinente sur le plan clinique. Tous les patients sauf un ont connu une amélioration moyenne de $12 \mathrm{D}$ après la TV, et cette amélioration était cliniquement significative dans 41 cas (91 \%) (tableau 2).

\section{Effet de la durée du traitement}

Étant donné que les patients ont suivi entre 5 et 20 séances de TV, nous voulions comparer les mesures du fonctionnement oculomoteur parmi les cohortes de patients ayant suivi de 5-10, de 12-17 ou 20 séances de TV (tableau 3). En général, des améliorations dans les mesures du fonctionnement oculomoteur ont été observées indépendamment de la durée du traitement. Toutefois, les patients qui ont suivi 20 séances ont montré une amélioration plus marquée des scores que les patients ayant suivi 10 séances de TV ou moins. Les notions suivantes étaient nettement meilleures avec une durée de traitement plus longue : l'amélioration de la flexibilité de vergence, la stéréopsie à distance et les mesures de FVP et de FVN, tant à distance qu'à proximité (tableau 3). De plus, les améliorations des résultats mesurés ont été comparées entre les groupes d’âge, et aucune tendance n’a été observée.

\section{DISCUSSION}

L'efficacité de la TV dans le traitement des symptômes liés au SPC du système visuel a été étudiée rétrospectivement. Dans l'ensemble, 56 patients ont été inclus dans la présente étude, et tous avaient une déficience clinique d'au moins une mesure du fonctionnement oculomoteur (tableau 1; tableau 2). À la suite de la TV, tous les patients ont montré des améliorations statistiquement ou cliniquement significatives dans au moins une mesure du fonctionnement oculomoteur liée au SPC (tableau 2). En général, les améliorations les plus importantes des mesures du fonctionnement oculomoteur ciblaient le PCP, la flexibilité de vergence et la flexibilité d'accommodation (tableau 2). Ces résultats étaient semblables à ceux de Ciuffreda et al., qui ont signalé que, dans de multiples études, 87 à $100 \%$ des patients ayant subi une lésion cérébrale acquise ont connu une réduction du dysfonctionnement d'accommodation après la TV. Plus récemment, Thiagarajan et Ciuffreda ont démontré l'efficacité de la TV pour améliorer l'amplitude de vergence et l'accommodation, la précision des mouvements oculaires saccadiques et la lecture globale chez les patients atteints d'un traumatisme cérébral léger. ${ }^{18}$ En outre, les améliorations des scores pour le PCP, la FVP à proximité et à distance, et les scores pour le CISS sur les symptômes étaient semblables à ceux observés chez les enfants et chez une cohorte dont la fourchette d'âge était semblable à celle de l'étude. ${ }^{22,24}$

Un fait intéressant est que les patients qui ont suivi 20 séances de TV avaient de meilleurs résultats (aussi moins variables) que ceux qui ont suivi entre 5 et 10 séances (tableau 3). Ces résultats sont semblables à ceux du groupe Convergence Insufficiency Treatment Trial Study, qui a observé que 12 semaines de séances de TV n'étaient pas suffisantes pour normaliser les mesures du fonctionnement oculomoteur chez les enfants atteints d'un traumatisme crânien léger. Toutefois, il est important de noter que, quelle que soit la durée du programme, les mesures du fonctionnement oculomoteur, comme la convergence et l'accommodation, sont susceptibles de s'améliorer en aussi peu que deux à six semaines de $\mathrm{TV}^{4,27}$

On a constaté que la thérapie visuelle réduisait les symptômes de l'inconfort visuel chez les patients avec un SPC, comme le démontrent les améliorations moyennes de $50 \%$ des scores du CISS, semblables aux résultats antérieurs déclarés par Scheiman et Wick (tableau 2). Toutefois, bien que des améliorations aient été constatées dans le fonctionnement oculomoteur, aucun résultat lié au retour au travail ou aux activités de la vie quotidienne n'a été enregistré dans cette étude, ce qui limite l'interprétation des résultats. La prudence est recommandée dans l'interprétation de ces résultats. En effet, une tendance se dessine pour les résultats oculomoteurs mesurés, particulièrement dans le cas du point de convergence proche, qui s'améliore au fil du temps dans les groupes placebo. ${ }^{27}$ Par ailleurs, les effets de la motivation des patients et des encouragements qu'ils ont reçus sur les résultats ne doivent pas être ignorés. ${ }^{27}$

\section{CONCLUSION}

Les résultats de cette analyse rétrospective appuient les conclusions d'études antérieures et montrent des améliorations statistiquement et cliniquement significatives du fonctionnement oculomoteur mesuré chez les patients avec des déficiences oculomotrices liées à un SPC, et ils appuient la TV comme option de traitement viable pour traiter les symptômes relatifs. De plus, les résultats actuels suggèrent que des durées de traitement plus longues (20 séances de TV) pourraient améliorer les résultats mesurés comparativement aux patients qui suivent 10 séances de TV ou moins. Toutefois, l'impact du traitement différé de la TV demeure incertain. Même s'il n'y avait pas de groupe placebo dans le cadre de cette étude, les résultats montrent des améliorations importantes au niveau des résultats mesurés pour tous les patients ayant suivi la TV et appuient la TV comme option de traitement pour le SPC. • 


\section{REMERCIEMENTS}

Les auteurs souhaitent remercier toute l'équipe de l'Okanagan Vision Therapy Optometry de l'engagement qu'elle prend d'assurer des programmes de thérapie visuelle et de réadaptation de grande qualité au sein de notre collectivité.

Nous tenons également à remercier Vision Therapy Canada, le COVD et l'OEPF des efforts inlassables qu'ils mènent pour faire progresser la qualité de la thérapie visuelle dans les cliniques du monde entier.

\section{AVIS DE NON-RESPONSABILITÉ.}

Les opinions exprimées dans l'article soumis sont les nôtres et non la position officielle d'une institution ou d'un bailleur de fonds.

\section{DIVULGATIONS FINANCIÈRES}

Cette étude a été autofinancée à l'aide de données cliniques et de ressources provenant de notre clinique - Okanagan Vision Therapy, Kelowna (C.-B.), Canada.

\section{CONFLITS D'INTÉRÊTS}

Les auteurs n'ont aucun conflit d'intérêts à signaler.

\section{DÉCLARATION DE PUBLICATION}

Cet article n'a pas été soumis/publié ailleurs.

COURRIEL DE L'AUTEUR CORRESPONDANT

Paul Rollett, OD, FCOVD, Okanagan Vision Therapy Optometry

drrollett@okanaganvisiontherapy.ca

Tableau 1 : Statistiques sommaires du régime de traitement et statistiques descriptives des patients recevant des traitements de thérapie visuelle pour le syndrome de post-commotion $(n=56)$.

\begin{tabular}{|l|c|c|c|}
\hline Mesure & Moyenne & Écart-type & Portée \\
\hline Âge & 42,6 & 13,8 & 12 à 62 \\
\hline Mois depuis la lésion & 15,1 & 14,2 & 2 à 60 \\
\hline $\begin{array}{l}\text { Retard dans le début de la TV (mois) } \\
\text { après l'évaluation }\end{array}$ & 1,5 & 2 & 0 à 12 \\
\hline $\begin{array}{l}\text { Séances de TV } \\
\text { Durée du programme (semaines) }\end{array}$ & 13,8 & 4,7 & 5 à 20 \\
\hline
\end{tabular}


Tableau 2 : Statistiques sommaires des données optométriques après le traitement de la thérapie visuelle pour le syndrome de post-commotion $(n=56)$.

\begin{tabular}{|c|c|c|c|c|c|c|c|}
\hline \multirow[t]{2}{*}{ Mesure } & \multicolumn{2}{|c|}{ Pré-TV } & \multicolumn{2}{|c|}{ Post-TV } & \multicolumn{3}{|c|}{ Amélioration du score } \\
\hline & moy. & type & moy. & type & moy. & type & portée \\
\hline $\mathrm{PCP}(\mathrm{cm})$ & 13,0 & 9,3 & $3,57^{*}$ & 1,94 & $65 \%$ & 2 & 6,2 à $97 \%$ \\
\hline Flexibilité de vergence (cpm) & 2,38 & 3,92 & $13,1^{*}$ & 3,18 & $83 \%$ & 27 & 0 à $100 \%$ \\
\hline $\begin{array}{l}\text { Flexibilité d'accommodation - } \\
\text { binoculaire }(\mathrm{cpm}) \delta\end{array}$ & 3,14 & 3,06 & $10,6^{*}$ & 1,87 & $71 \%$ & 26 & 17 à $100 \%$ \\
\hline King-Devick (s) & 76,4 & 41,0 & $51,4^{*}$ & 18,3 & $27 \%$ & 16 & 0 à $73 \%$ \\
\hline Stéréoscopique - 40 cm (secondes d'arc) & 79,8 & 40,6 & $42,6^{*}$ & 14,3 & $39 \%$ & 23 & 0 à $85 \%$ \\
\hline Stéréoscopique - 6 mètres (secondes d'arc) & 134 & 125 & $48,2^{*}$ & 12,8 & $41 \%$ & 31 & 0 à $90 \%$ \\
\hline CISS (score) & 38,2 & 11,42 & $20,0^{*}$ & 8,00 & $49 \%$ & 14 & 23 à $100 \%$ \\
\hline \multirow[t]{2}{*}{ Mesure } & \multicolumn{2}{|c|}{ Pré-TV } & \multicolumn{2}{|c|}{ Post-TV } & \multicolumn{3}{|c|}{ Amélioration du score } \\
\hline & moy. & type & moy. & type & moy. & type & portée \\
\hline Rupture de la FVP - D (6 m) & 8,05 & 5,94 & $19,4^{*}$ & 7,43 & 11,4 & 7,4 & 0 à 30 \\
\hline Rétablissement de la FVP - D (6 m) & 4,36 & 4,86 & $14,8^{*}$ & 6,67 & 10,4 & 6,4 & 0 à 28 \\
\hline Rupture de la FVN - D (6 m) & 3,77 & 2,36 & $8,46^{*}$ & 2,98 & 4,7 & 3,3 & 0 à 16 \\
\hline Rétablissement de la FVN -D (6 m) & 1,09 & 2,51 & $5,96^{*}$ & 2,27 & 4,9 & 3 & 0 à 15 \\
\hline Rupture de la FVP - D $(40 \mathrm{~cm})$ & 11,4 & 7,25 & $29,6^{*}$ & 6,98 & 18,2 & 8,9 & 0 à 38 \\
\hline Rétablissement de la FVP - D $(40 \mathrm{~cm})$ & 4,46 & 6,16 & $21,3^{*}$ & 7,49 & 16,9 & 9,1 & 0 à 38 \\
\hline Rupture de la FVN - D (40 cm) & 11,1 & 5,99 & $21,4^{*}$ & 3,30 & 10,3 & 6,3 & 0 à 27 \\
\hline Rétablissement de la FVN - D $(40 \mathrm{~cm})$ & 6,48 & 5,37 & $16,8^{*}$ & 3,45 & 10,3 & 6,2 & -4 à 22 \\
\hline
\end{tabular}

*Amélioration importante du score $(\mathrm{p}<0,001)$.

$\delta n=22$, âge moyen de $28,4 \pm 10,1$ 
Tableau 3 : Statistiques sommaires des améliorations des scores des patients à la suite de traitements de thérapie visuelle pour le syndrome de post-commotion regroupées selon le nombre de séances de $T V(n=56)$.

\begin{tabular}{|c|c|c|c|c|c|c|c|c|}
\hline Nombre de séances de TV & \multicolumn{2}{|c|}{5 à 20} & \multicolumn{2}{|c|}{5 à 10} & \multicolumn{2}{|c|}{12 à 17} & \multicolumn{2}{|c|}{20} \\
\hline \multirow[t]{2}{*}{ Dénombrement (n) } & \multicolumn{2}{|c|}{56} & \multicolumn{2}{|c|}{29} & \multicolumn{2}{|c|}{10} & \multicolumn{2}{|c|}{17} \\
\hline & moy. & type & moy. & type & moy. & type & moy. & type \\
\hline Mois depuis la lésion & 15,1 & 14,2 & 14,3 & 13,2 & 14,9 & 18,6 & 16,4 & 13,7 \\
\hline \multirow[t]{2}{*}{ Durée du programme (semaines) } & 15,2 & 4,86 & 11,2 & 2,13 & 16,0 & 1,56 & 21,5 & 0,94 \\
\hline & \multicolumn{8}{|c|}{ Amélioration du score en pourcentage } \\
\hline Mesure & moy. & type & moy. & type & moy. & type & moy. & type \\
\hline $\operatorname{PCP}(\mathrm{cm})$ & $65 \%$ & 20 & $67 \%$ & 18 & $64 \%$ & 25 & $62 \%$ & 21 \\
\hline Flexibilité de vergence (cpm) & $83 \%$ & 27 & $77 \%$ & 32 & $83 \%$ & 23 & $94 \%$ & 12 \\
\hline $\begin{array}{l}\text { Flexibilité d'accommodation } \\
(\mathrm{cpm}) \delta\end{array}$ & $71 \%$ & 26 & $66 \%$ & 27 & $85 \%$ & 17 & $72 \%$ & 31 \\
\hline King-Devick (s) & $27 \%$ & 16 & $23 \%$ & 16 & $30 \%$ & 12 & $31 \%$ & 19 \\
\hline Stéréoscopique - près & $39 \%$ & 23 & $34 \%$ & 22 & $54 \%$ & 27 & $38 \%$ & 20 \\
\hline Stéréoscopique - distance & $41 \%$ & 31 & $29 \%$ & 27 & $61 \%$ & 33 & $50 \%$ & 28 \\
\hline \multirow[t]{2}{*}{ CISS (score) } & $49 \%$ & 14 & $50 \%$ & 16 & $55 \%$ & 14 & $46 \%$ & 9 \\
\hline & \multicolumn{8}{|c|}{ Amélioration du score } \\
\hline Mesure & moy. & type & moy. & type & moy. & type & moy. & type \\
\hline Rupture de la FVP - D (6 m) & 11,4 & 7,42 & 10,8 & 8,08 & 10,0 & 5,25 & 13,1 & 7,36 \\
\hline Rétablissement de la FVP - D (6 m) & 10,4 & 6,44 & 10,3 & 7,43 & 9,80 & 4,57 & 10,9 & 5,81 \\
\hline Rupture de la FVN - D (40 cm) & 4,70 & 3,35 & 4,59 & 3,59 & 4,60 & 3,66 & 4,94 & 2,88 \\
\hline $\begin{array}{l}\text { Rétablissement de la FVN - D } \\
(40 \mathrm{~cm})\end{array}$ & 4,88 & 3,03 & 5,10 & 3,45 & 3,60 & 2,84 & 5,24 & 2,25 \\
\hline Rupture de la FVP - D (40 cm) & 18,2 & 8,92 & 17,2 & 8,56 & 16,6 & 10,8 & 20,6 & 8,42 \\
\hline $\begin{array}{l}\text { Rétablissement de la FVP - D } \\
(40 \mathrm{~cm}) \text { Près }\end{array}$ & 16,9 & 9,13 & 15,9 & 9,71 & 16,4 & 9,65 & 18,8 & 7,95 \\
\hline Rupture de la FVN - D (40 cm) & 10,3 & 6,33 & 9,66 & 5,10 & 9,60 & 6,31 & 11,8 & 8,14 \\
\hline $\begin{array}{l}\text { Rétablissement de la FVN - D } \\
(40 \mathrm{~cm})\end{array}$ & 10,3 & 6,17 & 9,93 & 5,08 & 8,90 & 7,34 & 11,8 & 7,17 \\
\hline
\end{tabular}

${ }^{\delta} \mathrm{n}=22$, âge moyen de $28,4 \pm 10,1$ 


\section{RÉFÉRENCES}

1. Mayo Clinic. Post-concussion syndrome. https://www.mayoclinic. org/diseases-conditions/post-concussion-syndrome/symptomscauses/syc-20353352 (accessed 5 June 2018).

2. Gallaway M, Scheiman M, Mitchell GL. Vision therapy for postconcussion vision disorders. Optom Vis Sci 2017 Jan;94(1):68-73.

3. Greenwald BD, Kapoor N, Singh AD. Visual impairments in the first year after traumatic brain injury. Brain Inj 2012;26(11):1338-59.

4. Skaler T, Niland P. Optometric vision therapy in rehabilitation of cognitive dysfunctions caused by traumatic brain injury. Scottish Intercollegiate Guidelines Network; 2015 https://www.acc.co.nz/ assets/research/f4d5ec9777/optometric-therapy-tbi-review.pdf (accessed 18 October 2019).

5. Broglio SP, Collins MW, Williams RM, Mucha A, Kontos AP. Current and emerging rehabilitation for concussion. a review of the evidence. Clin Sports Med 2015 Apr;34(2):213-31.

6. Lew HL, Pogoda TK, Baker E, et al. Prevalence of dual sensory impairment and its association with traumatic brain injury and blast exposure in OEF/OIF veterans. J Head Trauma Rehabil 2011 Nov-Dec;26(6):489-96.

7. Hartvigsen J, Boyle E, Cassidy JD, Carroll LJ. Mild traumatic brain injury after motor vehicle collisions: what are the symptoms and who treats them? A population-based 1-year inception cohort study. Arch Phys Med Rehabil 2014 Mar;95(3 Suppl):S286-94.

8. Dougherty AL, MacGregor AJ, Han PP, Heltemes KJ, Galarneau MR. Visual dysfunction following blast-related traumatic brain injury from the battlefield. Brain Inj 2011;25(1):8-13.

9. Bulson R, Jun W, Hayes J. Visual symptomatology and referral patterns for Operation Iraqi Freedom and Operation Enduring Freedom veterans with traumatic brain injury. J Rehabil Res Dev 2012;49(7):1075-82.

10. Ciuffreda KJ, Kapoor N, Rutner D, Suchoff IB, Han ME, Craig S. Occurrence of oculomotor dysfunctions in acquired brain injury: a retrospective analysis. Optometry 2007 Apr;78(4):155-61.

11. Master CL, Scheiman M, Gallaway M, et al. Vision diagnoses are common after concussion in adolescents. Clin Pediatr (Phila) 2016 Mar;55(3):260-7.

12. Porcar E, Martinez-Palomera A. Prevalence of general binocular dysfunctions in a population of university students. Optom Vis Sci 1997 Feb;74(2):111-3.

13. Scheiman M, Gwiazda J, Li T. Non-surgical interventions for convergence insufficiency. Cochrane Database Syst Rev 2011 Mar 16;(3):CD006768.
14. Lee SH, Moon BY, Cho HG. Improvement of vergence movements by vision therapy decreases K-ARS scores of symptomatic ADHD children. J Phys Ther Sci 2014 Feb;26(2):223-7.

15. Scheiman M, Wick B, eds. Clinical management of binocular vision: Heterophoric, accommodative, and eye movement disorders. Philadelphia: Lippincott, Williams \& Wilkins, 2008.

16. Ciuffreda KJ, Rutner D, Kapoor N, Suchoff IB, Craig S, Han ME. Vision therapy for oculomotor dysfunctions in acquired brain injury: a retrospective analysis. Optometry 2008 Jan;79(1):18-22.

17. Thiagarajan P, Ciuffreda KJ. Effect of oculomotor rehabilitation on vergence responsivity in mild traumatic brain injury. J Rehabil Res Dev 2013;50(9):1223-40.

18. Thiagarajan P, Ciuffreda KJ. Versional eye tracking in mild traumatic brain injury (mTBI): Effects of oculomotor training (OMT). Brain Inj 2014;28(7):930-43.

19. Thiagarajan P, Ciuffreda KJ. Effect of oculomotor rehabilitation on accommodative responsivity in mild traumatic brain injury. $\mathrm{J}$ Rehabil Res Dev 2014;51(2):175-91.

20. Thiagarajan P, Ciuffreda KJ, Capo-Aponte JE, Ludlam DP, Kapoor $\mathrm{N}$. Oculomotor neurorehabilitation for reading in mild traumatic brain injury (mTBI): An integrative approach. NeuroRehabilitation 2014;34(1):129-46.

21. Alvarez TL, Jaswal R, Gohel S, Biswal BB. Functional activity within the frontal eye fields, posterior parietal cortex, and cerebellar vermis significantly correlates to symmetrical vergence peak velocity: An ROI-based, fMRI study of vergence training. Front Integr Neurosci 2014 Jun 17;8:50.

22. Lacroix Z, Leat SJ, Christian LW. Role of primary care optometrists in the assessment and management of patients with traumatic brain injuries in Canada. Can J Optom 2016;80(1):13-7.

23. Elliott BD, ed. Clinical procedures in primary eye care, 3 rd edn. Philadelphia, Elsevier Butterworth-Heinemann, 2007.

24. Convergence Insufficiency Treatment Trial Study Group. Randomized clinical trial of treatments for symptomatic convergence insuf ficiency in children. Arch Ophthalmol 2008 Oct;126(10):1336-49.

25. Press LJ. Applied concepts in vision therapy. Optometric Extension Program Fndtn; OEP Edition, 1997.

26. Rosner B, Glynn RJ, Lee ML. The Wilcoxon signed rank test for paired comparisons of clustered data. Biometrics 2006 Mar;62(1):185-92.

27. Horwood AM, Toor SS, Riddell PM. Change in convergence and accommodation after two weeks of eye exercises in typical young adults. J AAPOS 2014 Apr;18(2):162-8. 